\title{
IMPACT OF AI IN INTERNET OF MEDICAL THINGS FOR HEALTH CARE DELIVERY
}

\author{
Neeta Nathani ${ }^{1} \square$, Zohaib Hasan ${ }^{2 \square}$ \\ ${ }^{1}$ Associate Professor, Gyan Ganga Institute of Technology and Sciences, Jabalpur (M.P.), India \\ ${ }^{2}$ Assistant Professor, Gyan Ganga Institute of Technology and Sciences, Jabalpur (M.P.), India
}

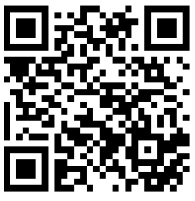

Received 22 July 2021

Accepted 05 August2021

Published 20 August 2021

\section{Corresponding Author}

Neeta Nathani,neeta_nathani@yahoo.com

DOI 10.29121/ijetmr.v8.i8.2021.1012

Funding: This research received no specific grant from any funding agency in the public, commercial, or not-for-profit sectors.

Copyright: (C) 2021 The Author(s). This is an open access article distributed under the terms of the Creative Commons Attribution License, which permits unrestricted use, distribution, and reproduction in any medium, provided the original author and source are credited.

\section{INTRODUTION}

Artificial intelligence (AI) has the potential to revolutionize the medical and healthcare industries. The impact of AI in medical diagnostics, in particular, has broadened the possibilities for providing high-quality patient care. One of the primary applications where AI providers are researching game-changing potential is early illness diagnosis, and they are fiercely investing in implementing $\mathrm{AI}$ in radiology to augment conventional techniques. In the AI in medical diagnostics business, picture recognition is likely the most attractive consumer offer. In millions of photos extracted from 
the target population, algorithms have been learning, unlearning, and relearning illness indicators. Outsourcing things to emerging economies has become popular as a cost-cutting strategy.

The use of devices is expanding, and the amount of data created as a result, as more devices are authorized and even approved by authorities such as the Federal Drug Administration (FDA) in the United States. The data is useful not only for treating chronic conditions or as part of an at-home post-operative care solution, but also for researchers, pharmaceutical product developers, other scientists, and health insurance companies, who can use it to better understand patterns and collaborate with institutions and professionals to improve care protocols so that the cost of care can be reduced and it shall be accessible to all.

The application of AI analytics becomes much more intriguing and beneficial when Internet of Mdical Things (IoMT) devices create huge data. For instance, Barry Solomon, co-founder of TeleMedCo, a technology business that pioneered the use of $\mathrm{AI}$ in emergency rooms (using IBM Watson's AI engine), and has now expanded into telemedicine solutions and services for seniors and veterans in the United States. It combines traditional "virtual visits" with remote patient monitoring via PrimoCare's gadgets and a 5G-connected hub.

Health monitoring will become less invasive and more automated as a result of smarter sensors, software, and services, lowering the need for human caretakers while restoring dignity to elders who have lost it through time. Wearables that target everything from swallowing problems to incontinence are now available from health startups. Ten years ago, monitoring a senior for hip-breaking falls might have been impractical without the help of a relative or personal nurse, but falls can now be detected and addressed immediately with smartwatches; similarly, wearables targeting everything from swallowing problems to incontinence were impractical without the help of a relative or personal nurse. Monitoring without wearables wireless devices that reduce or remove human involvement in the monitoring process and medically focused IoMT sensors developed specifically to record human biometrics will be the next steps.

\section{INTRODUCTION TO INTERNET OF MEDICAL THINGS (IOMT)}

The value of medical IoT (IoMT) is being magnified by the synergistic expansion of machine learning (ML) and artificial intelligence. In processing vast amounts of continually streaming data from linked medical devices, doctors are able to derive actionable findings more rapidly and reliably.

The medical field is no exception to the growth of IoT applications across industries. Metrics and patient feedback have become critical for care providers as many healthcare system shifts to evidence-based outcomes with incentives that are beginning to match. Payers are becoming increasingly interested in lowering costs by using more effective therapies.

Data-driven care will continue to expand and will have a positive impact on both treatment costs and quality. Healthcare has improved its ability to steer the ship in that direction over the last decade. The main benefit of sensors in healthcare is that they shorten the time between measurement, detection, and treatment. Insulin pumps use blood glucose monitors with sensors under the skin that communicate blood glucose levels to external receivers to measure and distribute 
doses at the proper moment, albeit with patient input. Furthermore, the data analytics tools currently available add context and meaning to measurements at a considerably faster rate than previously conceivable.

\section{ARTIFICIAL INTELLIGENCE (AI) IN MEDICAL DIAGNOSTICS KEY TRENDS}

Deep learning algorithms have sparked a flurry of activity in order to improve access to high-quality diagnostics at a reasonable cost. This could save a person's life if they have a chronic illness that is not diagnosed in a timely manner. Patients who are on the receiving end are individuals who are at high risk for cardiovascular disease. Another factor driving AI research in the medical diagnostics market is the need to improve diagnosis accuracy, whether through a single image or a series of images.

As seen by the growth of AI-based apps, the internet of medical things is making significant progress. On the one hand, AI businesses want to close the huge gap between demand and availability of radiologists, while also lowering the cost of radiological scans. The use of $\mathrm{AI}$ apps in the detection of diseases and increase patient-centered care is, in general, the key clinical proposition of $\mathrm{AI}$ in healthcare.

Origin Wireless has created a "wireless AI" system that maps closed environments using Wi-Fi signals. The wireless radio waves in a room generate an unseen "wave pool," which Origin's Remote Patient Monitoring system monitors for ripples that indicate disruptions. Origin RPM detects when a person quickly transitions from standing to laying on the floor without the need of a camera or motion sensors, and can send a warning to nearby caretakers or off-site family members. Subtle variations in data streams can reveal granular changes in a person's activity, breathing, and sleeping patterns. SakuraTech, a Japanese firm, is employing millimetre wave signals to wirelessly monitor up to four heart and respiration rates at the same time, promising to operate over common obstacles like clothing and blankets while transferring data to the AWS cloud for continuous remote monitoring.

It would be impossible to understand room-scale, volumetric quantities of wireless data in this way without machine learning, similar to a sonar system constantly seeing moving objects in the water without determining their intent. However, trained AI can comprehend a room's layout as depicted by radio waves, then identify dangerously unusual patterns in the humans who dwell there, all without invading personal privacy. Wi-Fi and millimetre wave scanning, unlike AI image segmentation, act like radar, and their data may be utilized to discern patterns without the requirement for photos or videos.

Essence Group just released 5G PERS, a senior independent living solution that includes activity monitoring, fall detection, and voice communication. 5G PERS monitors a collection of typical IoT motion sensors, but it employs $5 \mathrm{G}$ cellular connectivity for infrastructure rather than Wi-Fi or 4G. PERS $5 \mathrm{G}$ can operate in homes without Wi-Fi routers because it connects IoT sensors to the cloud over a cellular connection - the solution is freestanding, so it can be installed and remotely monitored without requiring the senior to maintain separate hardware or services.

General-purpose IoT sensors have been utilized to enable everything from smart refrigerators to industrial quality assurance systems using cameras and movement detectors, but medically oriented IoMT sensors connect wirelessly to 
health clouds for individual biometric monitoring and data storage. IoMT sensors can be significantly more "personal" than ever before because they're created exclusively for sensing individual human life signals: Their tiny electronics can enable external motion tracking in always-on wearables, as well as internal monitoring via ingestible wireless pills like HQ, Inc.'s CorTemp, a core temperature gauge that stays within your body for 24-36 hours.

The trend is clear: IoMT sensors will continue to improve in terms of power, ease of use, and widespread adoption. Even smaller 3-nanometer chips will be commercially available next year, making microchipped pills literally easier to swallow. New 5-nanometer chip fabrication has already yielded atomic-scale transistors that can be powered by barely any energy, and even smaller 5nanometer chips will be commercially available next year. Simultaneously, mobile AI processors are roughly doubling in performance every year, implying that tomorrow's client devices may have AI capabilities that are superior to yesterday's cloud and edge servers. Two years from now, remote monitoring tasks that seemed impossible two years ago would appear to be completely within the capabilities of even the most basic handsets.

\section{CATEGORIZING MEDICAL IOT APPLICATIONS}

1) Diagnostics: Devices that track bodily metrics that may signal medical diseases such as diabetes and atrial fibrillation are becoming more common. Continuous monitoring of vital physiological factors such as blood chemistry, blood pressure, brain activity, and pain levels is possible. This can aid in the detection of early indicators of illness start or activity, resulting in better responses. Once a disease propensity or risk factor has been established, causal indications can be closely tracked with the correct focused sensors. Because of features like heart rhythm monitoring and fall detection, even the most recent version of Apple Watch 4 has been classified as a class 2 medical equipment. It should be noted that the majority of consumer-oriented devices have not gone through the FDA regulatory procedure to become medical devices.

2) Recuperation: Patients' postoperative recovery time accounts for a large portion of the operation cost, and reducing it is a critical component of cost reduction. For a total knee replacement, for example, hospitalization in the United States takes roughly two days compared to four to five days in the United Kingdom's National Health Service (NHS). There is a need to cut down on time spent in a skilled nursing facility and physiotherapy outside of the hospital. This can be performed by using wearable sensors to help with exercise, compliance, and remote monitoring for issues that could lead to changes if not addressed in a timely manner.

Sensors can monitor a variety of vital indicators and alert caregivers to intervene in a timely manner. Sensors in conjunction with telemedicine make it even easier to assist in the rehabilitation process. Knowing what patients are up to in between visits can assist shorten the time it takes for them to recuperate from surgical operations. Indeed, a three-year collaboration between Geisinger System and Force Therapeutics led in much better outcomes. According to Greg Slabodkin of Health Data Management, this includes a 30 percent reduction in hospital length of stay, a 56 percent drop in skilled nursing facility utilisation, and an 18 percent reduction in readmissions. 
3) Chronic Care: With blood pressure, glucose levels, sweat, and even tear analysis, sensors that detect body parameters are becoming increasingly sophisticated. When compared to standardized examinations, the benefit is not just in terms of logistics, but also in terms of data collection frequency. In chronic degenerative conditions like rheumatoid arthritis, mobility sensors can aid improve stride and form. The monitoring and response of patients to treatment compliance is another category of IoMT device application. Poor outcomes and prolonged recovery can be avoided in chronic care by using IoT devices for measurement and monitoring.

4) Prophylactics: Devices that actively engage patients with guided exercise can help reduce injury and the related expenditures of medical treatment. Examples of how devices can aid in prevention include joint range of motion in the orthopaedic area and posture alignment to prevent cervical spondylosis. Upright is an example of a device.

Wearables, for example, may help the elderly avoid catastrophic falls by monitoring their activities and detecting anything unexpected that could lead to a loss of balance and a fall. The inbuilt IMU (Inertial Measurement Unit) in Apple's watch detects a fall or the likelihood of one. It can even be used to measure tremors caused by neurological illnesses such as Parkinson's disease.

\section{CONCLUSION}

The synergistic expansion of machine learning (ML) and artificial intelligence is increasing the usefulness of medical IoT. Data analytics and machine learning (ML) help speed up the treatment process by processing enormous amounts of continually streaming data from sensor-assisted medical devices. Preventive treatment, using streaming data, can drastically minimize hospitalization and the expense of acute care. This would boost productivity while also improving patient happiness and results. However, some data security concerns in transit and at rest must be carefully assessed. Furthermore, the possibility of false positive readings might put patients and the healthcare system under undue stress. The three key features of IoMT that must constantly be prioritized are accuracy, repeatability, and reliability.

\section{REFERENCES}

Ashique K, Kaliyadan F, Aurangabadkar SJ. (2015) Clinical photography in dermatology using smartphones: an overview. Indian Dermatol Online J ;6(3):158-163. Retrieved from https://dx.doi.org/10.4103\%2F22295178.156381

Baur K, Schättin A, de Bruin ED, Riener R, Duarte JE, Wolf P. (2018) Trends in robotassisted and virtual reality-assisted neuromuscular therapy: a systematic review of health-related multiplayer games. J Neuroeng Rehabil Nov 19;15(1):107. Retrieved from https://doi.org/10.1186/s12984-018-04499

Bhelonde A, Didolkar N, Jangale S, Kulkarni N. (2015) Flexible Wound Assessment System for Diabetic Patient Using Android Smartphone. In: International Conference on Green Computing and Internet of Things. 2015 Presented at: 
ICGCIoT'15; $\quad$ October 8-10; $\quad$ Noida, India. $\quad$ Retrieved from https://doi.org/10.1109/ICGCIoT.2015.7380509

Birckhead B, Khalil C, Liu X, Conovitz S, Rizzo A, (2019) Danovitch I, et al. Recommendations for methodology of virtual reality clinical trials in health care by an international working group: iterative study. JMIR Ment Health Jan 31;6(1):e11973.

Chen HS, Jarrell JT, Carpenter KA, Cohen DS, Huang X. (2019) Blockchain in healthcare: a patient-centered model. Biomed J Sci Tech Res Aug 8;20(3):15017-15022. Retrieved from https://www.ncbi.nlm.nih.gov/pmc/articles/PMC6764776/

Chen S, Jones C, Moyle W. (2018) Social robots for depression in older adults: a systematic review. J Nurs Scholarsh Nov;50(6):612-622. Retrieved from https://doi.org/10.1111/jnu.12423

Chirico A, Lucidi F, De Laurentiis M, Milanese C, Napoli A, Giordano A. (2016) Virtual reality in health system: beyond entertainment. A mini-review on the efficacy of VR during cancer treatment. J Cell Physiol Feb;231(2):275-287. Retrieved from https://doi.org/10.1002/jcp.25117

Dang LM, Piran MJ, Han D, Min K, Moon H. (2019) A survey on internet of things and cloud computing for healthcare. Electronics Jul 9 ;8(7) :768. Retrieved from https://doi.org/10.3390/electronics8070768

Darwish A, Hassanien AE, Elhoseny M, Sangaiah AK, Muhammad K. (2017) The impact of the hybrid platform of internet of things and cloud computing on healthcare systems: opportunities, challenges, and open problems. J Ambient Intell Human Comput Dec 29;10(10):4151-4166. Retrieved from https://doi.org/10.1007/s12652-017-0659-1

De Carvalho M, Dias T, Duchesne M, Nardi A, Appolinario J. (2017) Virtual reality in health system: beyond entertainment. A mini-review on the efficacy of VR during cancer treatment. Behav Sci (Basel) Jul 9;7(3). Retrieved from https://doi.org/10.1002/jcp.25117

Dojchinovski D, Ilievski A, Gusev M. (2019) editors. Interactive home healthcare system with integrated voice assistant 2019;. Retrieved from https://doi.org/10.23919/MIPR0.2019.8756983

Doryab A, Villalba DK, Chikersal P, Dutcher JM, Tumminia M, Liu X, et al. (2019) Identifying behavioral phenotypes of loneliness and social isolation with passive sensing: statistical analysis, data mining and machine learning of smartphone and FitBit data. JMIR Mhealth Uhealth Jul 24;7(7):e13209.

Eckert M, Volmerg JS, Friedrich CM. (2019) Augmented reality in medicine: systematic and bibliographic review. JMIR Mhealth Uhealth Apr 26;7(4):e10967.

Fisk M, Livingstone A, Pit SW. (2020) Telehealth in the context of covid-19: changing perspectives in Australia, the United Kingdom, and the United States. J Med Internet Res Jun 9;22(6):e19264.

GBD (2017) Disease Injury Incidence Prevalence Collaborators. Global, regional, and national incidence, prevalence, and years lived with disability for 354 diseases and injuries for 195 countries and territories, 1990-2017: a systematic analysis for the Global Burden of Disease Study 2017. Lancet 


$\begin{array}{cccccc}2018 \quad \text { Nov 10; } & \text { 392(10159):1789-1858. } & \text { Retrieved } & \text { from } \\ \text { https://doi.org/10.1016/S0140-6736(18)32279-7 } & & \end{array}$

Gerup J, Soerensen CB, Dieckmann P. (2020) Augmented reality and mixed reality for healthcare education beyond surgery: an integrative review. Int J Med Educ Jan 18;11:1-18. from https://dx.doi.org/10.5116\%2Fijme.5e01.eb1a

Ilievski A, Dojchinovski D, Gusev M. (2019) Interactive Voice Assisted Home Healthcare Systems. In: Proceedings of the 9th Balkan Conference on Informatics. 2019 Presented at: BCI'19; September 26-28,; University of Sofia, Bulgaria. Retrieved from https://doi.org/10.1145/3351556.3351572

Internet of Things (IoT) (2019) in Healthcare. Research Markets.. URL: https://www.medicaldevice-network.com/comment/bringing-internetthings-healthcare/ [accessed 2020-10-02].

Jadczyk T, Kiwic O, Khandwalla RM, Grabowski K, Rudawski S, Magaczewski P, et al. (2019) Feasibility of a voice-enabled automated platform for medical data collection: CardioCube. Int J Med Inform Sep;129:388-393. [CrossRef] [Medline] Retrieved from https://doi.org/10.1016/j.ijmedinf.2019.07.001

Kakria P, Tripathi NK, Kitipawang P. (2015) A real-time health monitoring system for remote cardiac patients using smartphone and wearable sensors. Int J Telemed Appl;2015:373474. Retrieved from https://doi.org/10.1155/2015/373474

Laranjo L, Dunn A, Tong H, Kocaballi A, Chen J, Bashir R, et al. (2018) Conversational agents in healthcare: a systematic review. J Am Med Inform Assoc Sep 1;25(9):1248-1258. $\quad$ Retrieved from https://doi.org/10.1093/jamia/ocy072

Levine DM, Ouchi K, Blanchfield B, Diamond K, Licurse A, Pu CT, et al. (2018) Hospital-level care at home for acutely ill adults: a pilot randomized controlled trial. J Gen Intern Med May;33(5):729-736. Retrieved from https://doi.org/10.7326/M19-0600

Li S, Xu LD, Zhao S. (2018) 5G internet of things: a survey. J Ind Inf Integration Jun;10:1-9. Retrieved from https://doi.org/10.1016/j.jii.2018.01.005

Lohse KR, Hilderman CG, Cheung KL, Tatla S, van der Loos HF (2014). Virtual reality therapy for adults post-stroke: a systematic review and meta-analysis exploring virtual environments and commercial games in therapy. PLoS One;9(3):e93318.

Retrieved

from https://doi.org/10.1371/journal.pone.0093318

Majumder S, Chen L, Marinov O, Chen C, Mondal T, Deen MJ. (2018) Noncontact wearable wireless ECG systems for long-term monitoring. IEEE Rev Biomed Eng;11:306-321.

Retrieved

from https://doi.org/10.1109/RBME.2018.2840336

Merchant R, Szefler SJ, Bender BG, Tuffli M, Barrett MA, Gondalia R, et al. (2018) Impact of a digital health intervention on asthma resource utilization. World Allergy Organ J;11(1):28 [FREE Full text] Retrieved from https://doi.org/10.1186/s40413-018-0209-0

Merchant RK, Inamdar R, Quade RC. (2016) Effectiveness of population health management using the propeller health asthma platform: a randomized clinical trial. J Allergy Clin Immunol Pract;4(3):455-463. https://doi.org/10.1016/j.jaip.2015.11.022 
Mitchell M, Kan L. (2019) Digital technology and the future of health systems. Health Syst Reform;5(2):113-120. Retrieved from https://doi.org/10.1080/23288604.2019.1583040

Mitchell-Box K, Braun KL. (2012) Fathers' thoughts on breastfeeding and implications for a theory-based intervention. J Obstet Gynecol Neonatal Nurs;41(6):E41-E50. Retrieved from https://doi.org/10.1111/j.15526909.2012.01399.x

Moyle W, Jones C, Cooke M, O'Dwyer S, Sung B, Drummond S. (2013) Social Robots Helping People With Dementia: Assessing Efficacy of Social Robots in the Nursing Home Environment. In: 6th International Conference on Human System Interactions (HSI). Presented at: HSI'13; June 6-8, 2013; Sopot, Poland. Retrieved from https://doi.org/10.1109/HSI.2013.6577887

Naik R, Macey N, West RJ, Godbehere P, Thurston SC, Fox R, et al. (2017) First use of an ingestible sensor to manage uncontrolled blood pressure in primary practice: the UK hypertension registry. J Community Med Health Educ;07(01).

Nazir S, Ali Y, Ullah N, García-Magariño I. (2019) Internet of things for healthcare using effects of mobile computing: a systematic literature review. Wireless Commun Mobile Comput 2019 Nov $14 ; 1-20$. Retrieved from https://doi.org/10.1155/2019/5931315

Pan J, McElhannon J. (2018) Future Edge Cloud and Edge Computing for Internet of Things Applications. IEEE Internet Things J Feb;5(1):439-449. Retrieved from https://doi.org/10.1109/JIOT.2017.2767608

Park YR, Lee E, Na W, Park S, Lee Y, Lee J. (2019) Is blockchain technology suitable for managing personal health records? Mixed-methods study to test feasibility. J Med Internet Res Feb 8;21(2):e12533.

Persky S. (2011) Application of virtual reality methods to obesity prevention and management research. J Diabetes Sci Technol Mar 1;5(2):333-339. Retrieved from https: //doi.org/10.1177\%2F193229681100500220

Plowman R, Peters-Strickland T, Savage G. (2018) Digital medicines clinical review on the safety of tablets with sensors.;17(9):849-852. Retrieved from https://doi.org/10.1080/14740338.2018.1508447

Połap D, Winnicka A, Serwata K, Kęsik K, Woźniak M. (2018) An intelligent system for monitoring skin diseases. Sensors (Basel) Aug 4;18(8). Retrieved from https://doi.org/10.3390/s18082552

Ritschel H, Seiderer A, Janowski K, Aslan I, André E. Drink-O-Mender (2018): An Adaptive Robotic Drink Adviser. In: Proceedings of the 3rd International Workshop on Multisensory Approaches to Human-Food Interaction. Presented at: MHFI'18; October 16, 2018; Boulder, Colorado. Retrieved from https://doi.org/10.1145/3279954.3279957

Saarikko T, Westergren UH, Blomquist T. (2017) The internet of things: are you ready for what's coming? Bus Horiz Sep ;60(5) :667-676. Retrieved from https://doi.org/10.1016/j.bushor.2017.05.010

Sangave NA, Aungst TD, Patel DK. (2019) Smart connected insulin pens, caps, and attachments: a review of the future of diabetes technology. Diabetes Spectr Nov;32(4):378-384. Retrieved from https://doi.org/10.2337/ds18-0069 
Sethi P, Sarangi S. (2017) Internet of things: architectures, protocols, and applications. J Electric Comput Eng. Retrieved from https://doi.org/10.1155/2017/9324035

Shimizu E, Ogawa Y, Yazu H, Aketa N, Yang F, Yamane M, et al. (2019) 'Smart Eye Camera': an innovative technique to evaluate tear film breakup time in a murine dry eye disease model. PLoS One;14(5):e0215130. Retrieved from https://doi.org/10.1371/journal.pone.0215130

Sposaro F, Tyson G. (2020:a) iFall: an Android application for fall monitoring and response. (1557-170X (Print)). Retrieved from https://doi.org/10.1109/IEMBS.2009.5334912

Tao V, Moy K, Amirfar VA (2016). A little robot with big promise may be future of personalized health care. Pharmacy Today Sep;22(9):38. Retrieved from https://doi.org/10.1016/j.ptdy.2016.08.022

Tashjian VC, Mosadeghi S, Howard AR, Lopez M, Dupuy T, Reid M, et al. (2017) Virtual reality for management of pain in hospitalized patients: results of a controlled trial. JMIR Ment Health Mar 29;4(1):e9. Retrieved from https://doi.org/10.1371/journal.pone.0219115

Torous J, Jän Myrick K, Rauseo-Ricupero N, Firth J. (2020) Digital mental health and covid-19: using technology today to accelerate the curve on access and quality tomorrow. JMIR Ment Health Mar 26;7(3):e18848.

Van der Putte D, Boumans R, Neerincx M, Rikkert M, de MM (2019). A Social Robot for Autonomous Health Data Acquisition Among Hospitalized Patients: An Exploratory Field Study. In: 14th ACM/IEEE International Conference on Human-Robot Interaction (HRI). Presented at: HRI'19; March 11-14, 2019; Daegu, Korea (South), Korea (South). Retrieved from https://doi.org/10.1109/HRI.2019.8673280

Valmaggia LR, Latif L, Kempton MJ, Rus-Calafell M (2016). Virtual reality in the psychological treatment for mental health problems: an systematic review of recent evidence. Psychiatry Res Feb 28;236:189-195. Retrieved from https://doi.org/10.1016/j.psychres.2016.01.015

Wan S, Gu Z, Ni Q. (2020) Cognitive computing and wireless communications on the edge for healthcare service robots. Comput Comm Jan;149:99-106. Retrieved from https://doi.org/10.1016/j.comcom.2019.10.012

Wu M, Lu T, Ling F, Sun J (2010). Research on the Architecture of Internet of Things. In: 3rd International Conference on Advanced Computer Theory and Engineering. Presented at: ICACTE'10; August 20-22, 2010; Chengdu, China. https://doi.org/10.1109/ICACTE.2010.5579493

Ye Q, Zhou J, Wu H. (2020) Using information technology to manage the covid-19 pandemic: development of a technical framework based on practical experience in China. JMIR Med Inform Jun 8;8(6):e19515.

Yin Y, Zeng Y, Chen X, Fan Y. (2016) The internet of things in healthcare : an overview. J Ind Inf Integration Mar;1:3-13. Retrieved from https://doi.org/10.1016/j.jii.2016.03.004 